\title{
Il controllo di gestione nella prospettiva relazionale e la sua evoluzione nelle aziende di servizi
}

\author{
Luciano Marchi
}

\section{Il controllo di gestione nella prospettiva relazionale}

Come è stato ben evidenziato nel primo numero di questa rivista, la collaborazione tra aziende è sempre più il percorso tipico per realizzare la crescita e determinare la strategia aziendale: dalle singole relazioni di partnership tra due aziende, alla rete di aziende, per arrivare all'azienda-rete. Parallelamente, si determina un ampliamento, oltre i confini della singola azienda, dell'orizzonte nell'ambito del quale formulare i giudizi di convenienza (Mancini, Management Control, n. 1/2011, p. 65).

L'esigenza di gestire le relazioni cooperative nella rete, monitorando e valutando i collegati processi di creazione di valore, determina dunque l'evoluzione del controllo di gestione nella prospettiva "relazionale" e "organizzativa".

Si realizza, in primo luogo, un ampliamento della dimensione spaziale del controllo alla rete di aziende con cui sviluppare relazioni cooperative, dal punto di vista organizzativo, coinvolgendo soggetti interni ed esterni all'azienda. La domanda a cui occorre dare risposta è pertanto: "Come riuscire a sviluppare un controllo di gestione che riesca a presidiare non solo i processi interni ma anche quelli esterni, le interrelazioni tra le unità della competizione/cooperazione, in un'ottica relazionale? Alcune indicazioni al riguardo sono fornite nel precedente editoriale della rivista (Marchi, Management Control, n. 3/2011, p. 10).

Si realizza, in secondo luogo, un ampliamento della dimensione organizzativa interna per gestire l'integrazione degli approcci formali con quelli informali del controllo di gestione.

Dal punto di vista teorico, cercando di individuare gli elementi di evoluzione dei sistemi di controllo parliamo di controllo organizzativo, magari con accezioni diverse, e di controllo relazionale. 
Nel primo articolo inserito nel presente numero della rivista (Pezzillo et al.) gli autori evidenziano come la regolazione delle diversità organizzative possa rafforzare la percezione delle identità individuali, dunque l'autoregolazione dei comportamenti e l'attivazione/rafforzamento del senso di commitment nei confronti dell'organizzazione.

Nel secondo articolo (Moro et al.) gli autori evidenziano, nella prospettiva del controllo relazionale, la rivisitazione dei contenuti e delle funzioni dei tradizionali strumenti di misurazione, affiancando approcci formali ed informali al controllo. Nell'articolo si analizza, in particolare, la complessa relazione che intercorre tra i Management Control Systems e la fiducia come meccanismo di controllo essenzialmente informale, riconoscendo sistemi di influenza reciproca particolarmente complessi.

\section{L'evoluzione del controllo di gestione nelle aziende di servizi}

L'evoluzione del controllo di gestione nell'ottica "organizzativa" e "relazionale" può assumere un grande rilievo nelle aziende di servizi, anche al fine di realizzare la necessaria integrazione interna-esterna in termini di rendicontazione e governance ampliata.

I caratteri evolutivi del controllo nelle aziende di servizi sono oggetto del terzo articolo presentato in questo numero della rivista (Cantele et al.) ma anche dei lavori che seguono realizzati nell'ambito del Gruppo di Studio e Attenzione AIDEA su "Politiche e management del Patrimonio Culturale nelle diverse prospettive del valore. Strumenti e metodi di misurazione e valutazione" a cura di Fabio Donato e Barbara Sibilio.

Le evidenze empiriche indicano una crescita degli strumenti di misurazione delle performance sia nelle Università che nelle Istituzioni culturali. Ma alcune riflessioni critiche si pongono a tale riguardo. In primo luogo, i sistemi di misurazione sono generalmente di tipo monodimensionale, senza considerare ulteriori ambiti rispetto a quello economico-finanziario, con approccio essenzialmente burocratico-formale. In secondo luogo, ci sentiamo di esprimere alcuni dubbi sui risultati di tali indagini empiriche e delle collegate rilevazioni statistiche che indicano elevati livelli di crescita dei sistemi informativi e del controllo di gestione, con il passaggio, ad esempio, dalla contabilità finanziaria alla contabilità economico-patrimoniale. I test di conformità da noi effettuati indicano infatti una diversa realtà: la nuova strumentazione non è correttamente utilizzata, non determina un controllo-guida, non riesce ancora ad incidere sulla cultura aziendale di molte delle realtà osservate. 